\title{
BMJ Open Insurance status, inhospital mortality and length of stay in hospitalised patients in Shanxi, China: a cross- sectional study
}

\author{
Xiaojun Lin, ${ }^{1}$ Miao Cai, ${ }^{1,2}$ Hongbing Tao, ${ }^{1}$ Echu Liu, ${ }^{2}$ Zhaohui Cheng, ${ }^{1}$ Chang Xu, ${ }^{1}$ \\ Manli Wang, ${ }^{1}$ Shuxu Xia, ${ }^{1}$ Tianyu Jiang ${ }^{1}$
}

To cite: Lin $\mathrm{X}$, Cai M, Tao $\mathrm{H}$, et al. Insurance status, inhospital mortality and length of stay in hospitalised patients in Shanxi, China: a crosssectional study. BMJ Open 2017;7:e015884. doi:10.1136/ bmjopen-2017-015884

- Prepublication history and additional material for this paper are available online. To view these files please visit the journal online (http://dx.doi. org/10.1136/bmjopen-2017015884).

Received 6 January 2017 Revised 9 June 2017 Accepted 12 June 2017

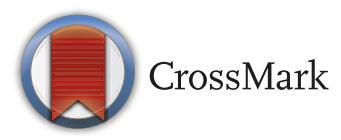

${ }^{1}$ Department of Health Administration, School of Medicine and Health Management, Tongji Medical College, Huazhong University of Science and Technology, Wuhan, Hubei, China

${ }^{2}$ Department of Health

Management and Policy, College for Public Health and Social Justice, Saint Louis University, St Louis, Missouri, USA

Correspondence to Professor Hongbing Tao; hhbtao@hust.edu.cn

\section{ABSTRACT}

Objectives To determine insurance-related disparities in hospital care for patients with acute myocardial infarction (AMI), heart failure (HF) and pneumonia.

Setting and participants A total of 22392 patients with AMI, 8056 patients with HF and 17161 patients with pneumonia were selected from 31 tertiary hospitals in Shanxi, China, from 2014 to 2015 using the International Classification of Diseases, Tenth Revision codes. Patients were stratified by health insurance status, namely, urban employee-based basic medical insurance (UEBMI), urban resident-based basic medical insurance (URBMI), new cooperative medical scheme (NCMS) and self-payment. Outcome measures Inhospital mortality and length of stay (LOS).

Results The highest unadjusted inhospital mortality rate was detected in NCMS patients independent of medical conditions $(4.7 \%, 4.4 \%$ and $11.1 \%$ for AMI, HF and pneumonia, respectively). The lowest unadjusted inhospital mortality rate and the longest LOS were observed in UEBMI patients. After controlling patient-level and hospitallevel covariates, the adjusted inhospital mortality was significantly higher for NCMS and self-payment among patients with $\mathrm{AMI}$, for NCMS among patients with HF and for URBMI, NCMS and self-payment among patients with pneumonia compared with UEBMI. The LOS of the URBMI, NCMS and self-payment groups was significantly shorter than that of the UEBMI group.

Conclusion Insurance-related disparities in hospital care for patients with three common medical conditions were observed in this study. NCMS patients had significantly higher adjusted inhospital mortality and shorter LOS compared with UEBMI patients. Policies on minimising the disparities among different insurance schemes should be established by the government.

\section{INTRODUCTION}

The Chinese government launched its new nationwide healthcare reform in 2009 to provide affordable and equitable access to healthcare. ${ }^{1}$ With advancements in this reform, the Chinese government has shown remarkable political will and commitment to universal health coverage. ${ }^{2}$ The current

\section{Strengths and limitations of this study}

- This comprehensive study explores the association between health insurance status and health outcome in Shanxi province in China, revealing the disparities among four insurance groups regarding adjusted inhospital mortality and length of stay for consideration for policy-makers.

- This study is based on a broad population comprising patients with acute myocardial infarction, heart failure and pneumonia.

- We are unable to capture all potential confounders in our study, especially the socioeconomic information of patients, because of the limited use of an administrative database.

- Patients in other levels of hospitals in Shanxi province, China, were not included in this study.

Chinese social health insurance (SHI) system consists of the urban employee-based basic medical insurance (UEBMI), urban resident-based basic medical insurance (URBMI) and the new cooperative medical scheme (NCMS). The coverage of SHI rose from $29.7 \%$ in 2003 to more than $95 \%$ of the Chinese population in $2011,{ }^{3}$ and this advancement was lauded as an 'unparalleled' achievement. $^{4}$

However, these three insurance schemes differ substantially in target population, administration, source of funding, funding level and benefit packages. ${ }^{15}$ Specifically, the UEBMI (initiated in 1994 and launched in 1998) is a mandatory programme targeting at urban employees and retired employees. ${ }^{4}$ In 2014, 283million urban employees were enrolled in the UEBMI. ${ }^{6}$ The annual premium of the UEBMI ( $8 \%$ of the payroll) is shared between employers and employees, where employers pay $6 \%$ as tax and employees pay $2 \%$. The per capita fund of UEBMI is approximately six and seven times higher 
than that of other two insurance programmes, reaching around $¥ 2840.6$ in $2014 .{ }^{6}$ The UEBMI provides the most comprehensive coverage, including both inpatient and outpatient care services, with the inpatient reimbursement rate of approximately $80 \%$ in 2014. Unlike the UEBMI, URBMI (initiated in 2007 and formally launched in 2009) is a voluntary programme aiming at covering urban residents who were not covered by the UEBMI. By the end of 2014, 314.5 million urban residents have participated in the URBMI. ${ }^{6}$ Its premium was highly subsidised by the government, while individuals contribute a small proportion. The per capita fund of URBMI was ¥524.4 in $2014 .^{7}$ The reimbursement rate for inpatient and outpatient care increased with time and reached $70 \%$ and $50 \%$, respectively, in 2014. ${ }^{8}$ Both UEBMI and URBMI funds were pooled at prefecture/municipality level and administered by the Ministry of Human Resource and Social Security. The NCMS was initiated in 2003 and formally launched in 2006. It is also a voluntary programme aiming to cover rural residents. By 2014, the NCMS has covered 736 million rural residents, accounting for $98.9 \%$ of the rural population. ${ }^{8}$ Similar to URBMI, the government subsidies are the major funding sources of NCMS, while the participants pay for a small proportion. The NCMS covered primarily inpatient care and some outpatient services for selected chronic conditions, and its reimbursement rate for inpatient and outpatient care reached $75 \%$ and $50 \%$ in 2014, respectively. NCMS was pooled at county level and managed by the Chinese National Health and Family Planning Commission. In China, type of registration (rural or urban) and employment status, rather than self-selection, are major determinants of insurance scheme enrolment. $^{9}$

In China's recent healthcare reform, universal health coverage has improved the accessibility to healthcare services, but this phenomenon does not guarantee equality in health outcome and healthcare utilisation of individuals enrolled in different insurance schemes. Recently, a few studies in China have explored the disparities in health outcome and healthcare utilisation across different health insurance groups, specifically among patients with acute myocardial infarction (AMI), ${ }^{11}{ }^{11}$ peritoneal dialysis, ${ }^{12}$ intracranial haemorrhage ${ }^{13}$ and schizophrenia. ${ }^{14}$ In 2005, $\mathrm{Yu}^{10}$ analysed the medical records of 4714 patients with AMI and found that insurance status was not associated with inhospital mortality significantly, whereas uninsured patients with AMI were less likely to receive interventions and medications and had a shorter length of stay (LOS). However, in a study exploring the association between medical insurances and clinical outcomes for patients with ST elevation myocardial infarction in Shanghai, Liu et $a l^{11}$ found that the incidence of major adverse events and cardiac mortality of patients with NCMS were higher than those of patients with other insurance types. Wang et $a l^{12}$ performed a single-centre study and demonstrated that the survival rate of NCMS patients in 5 years is lower than that of UEBMI patients who underwent peritoneal dialysis. Kong et $a l^{13}$ compared the differences of inhospital mortality and LOS by insurance types, and they found that patients locally insured had higher death rate and longer LOS than both non-locally insured and uninsured patients, but risk adjustment was not conducted in their study. Feng et $a l^{14}$ explored the impact of medical insurance policies on the hospitalisation service utilisation of patients with schizophrenia and revealed that patients with UEBMI were admitted to high-level medical institutions and received costly medications. Fang et $a l^{15}$ indicated that UEBMI respondents were more likely to receive preventive healthcare services than NCMS respondents. Liu et $a \theta^{\theta}$ found that UEBMI participants achieved better self-reported health status, physical functions and psychological well-being than URBMI, NCMS and uninsured participants did, using the data of the National Survey of the Aged Population in Urban/Rural China in 2006 and 2010.

Previous studies have indicated that there are insurance-related disparities in health outcomes and healthcare utilisation in several diseases or specific populations, but whether this relationship persists in a wider population or other diseases remains unknown. Moreover, the risk adjustment and the nest or cluster effects (ie, patients treated at the same hospital experience similar outcomes) were ignored in previous studies in China, which may result in biased estimations and provide wrong evidence in the policy formulation process.

This study proposes to explore the disparities among three SHI groups and self-payment group (ie, patients who pay the cost of care without reimbursement) regarding adjusted inhospital mortality and LOS to fill in the gap in the literature. The primary reason why we choose AMI, heart failure (HF) and pneumonia is that they are common conditions in China. Further, we can compare our results with previous studies that analysed patients with AMI. We used a large administrative database in Shanxi to examine this important question. Previous studies suggest that both NCMS and URBMI have lower funding level and more limited benefit packages than UEBMI and that NCMS participants have worse outcomes and utilise less healthcare services ${ }^{911}{ }^{12}$; hence, we hypothesised that NCMS and URBMI patients hospitalised with AMI, HF and pneumonia have higher adjusted inhospital mortality and shorter LOS than UEBMI patients.

\section{METHODS}

\section{Data source}

Shanxi province is located in Northern China. It has 36.5 million residents, with $53.79 \%$ living in urban areas in 2014. ${ }^{16}$ According to the statistics of the National Health and Family Planning Commission, Shanxi had 37 tertiary hospitals in 2014 and 2015, but the data in six tertiary hospitals were unavailable for the study team. Therefore, we only included 31 tertiary hospitals in Shanxi.

We conducted a retrospective study of patients hospitalised between 1 January 2014 and 31 December 2015 using administrative data from hospital electronic health 
records (EHRs). The data contain over 200 variables, including patients' sociodemographic characteristics (eg, age, gender, race/ethnicity, occupation and insurance status), diagnosis codes (ie, principal diagnosis code, up to 10 secondary diagnosis codes), up to seven procedure codes, total costs, service charges in subcategories, LOS and outcomes (such as discharge status and medical adverse events during the hospitalisation). The EHRs in those hospitals follow a national template and have adopted standardised disease coding in the International Classification of Diseases, Tenth Revision (ICD-10). The EHRs from various hospitals in Shanxi were entered by qualified coders who have received professional coding training and were certified by the Medical Record Management Association of the Chinese Hospital Association. In addition, the study team randomly sampled $10 \%$ of the EHRs to validate the accuracy of variables, such as patients' demographic characteristics, insurance type, principal and secondary diagnosis codes and LOS. The overall variable accuracy reached approximately $97 \%$. All patient, medical staff and hospital identifiers, such as name, ID card number, address, postcode and insurance number, were excluded before the data were provided to the study team.

\section{Patients}

We used ICD-10 codes with variations in the three digits after the decimal point. Then, we identified all patients with the principal diagnosis of AMI (ICD-10 codes: I21.x), HF (ICD-10 codes: I50.x) and pneumonia
(ICD-10 codes: J10.x-J18.x) between 1 January 2014 and 31 December 2015 (see online supplementary table $1)$. We chose the three diseases as the subject of this study for the following reasons. Cardiovascular disease (CVD) remains the leading cause of death in China, with mortality rates of $42.5 \%$ in urban areas and $44.6 \%$ in rural areas. ${ }^{17} \mathrm{AMI}$ and HF have been two common causes of hospitalisation in China. ${ }^{18} 19$ In 2014, 290 million patients were estimated to have CVD, and one in five Chinese adults suffered from CVD. Among individuals with CVD, 2.5 million had a myocardial infarction and 4.5 million had an $\mathrm{HF}^{20}$ Similarly, pneumonia is one of the leading causes of death in adults and children in China, ${ }^{21}$ and 2.5 million people were estimated to suffer from pneumonia annually and that $5 \%$ of them die of pneumonia-related diseases. ${ }^{22}$

Patients in the UEBMI, URBMI, NCMS and self-payment groups were selected for further analyses. As shown in figure 1, we excluded patients under 18 years and over 90 years of age and patients with missing health insurance data and discharge disposition. In addition, we excluded patients who were transferred to another hospital or community health service centres because their admissions were truncated and subsequent treatment information in other facilities was unavailable for our study. Furthermore, we excluded patients who were discharged alive within 1 day after admission because they were likely to leave against medical advice and the treatment time was very limited.

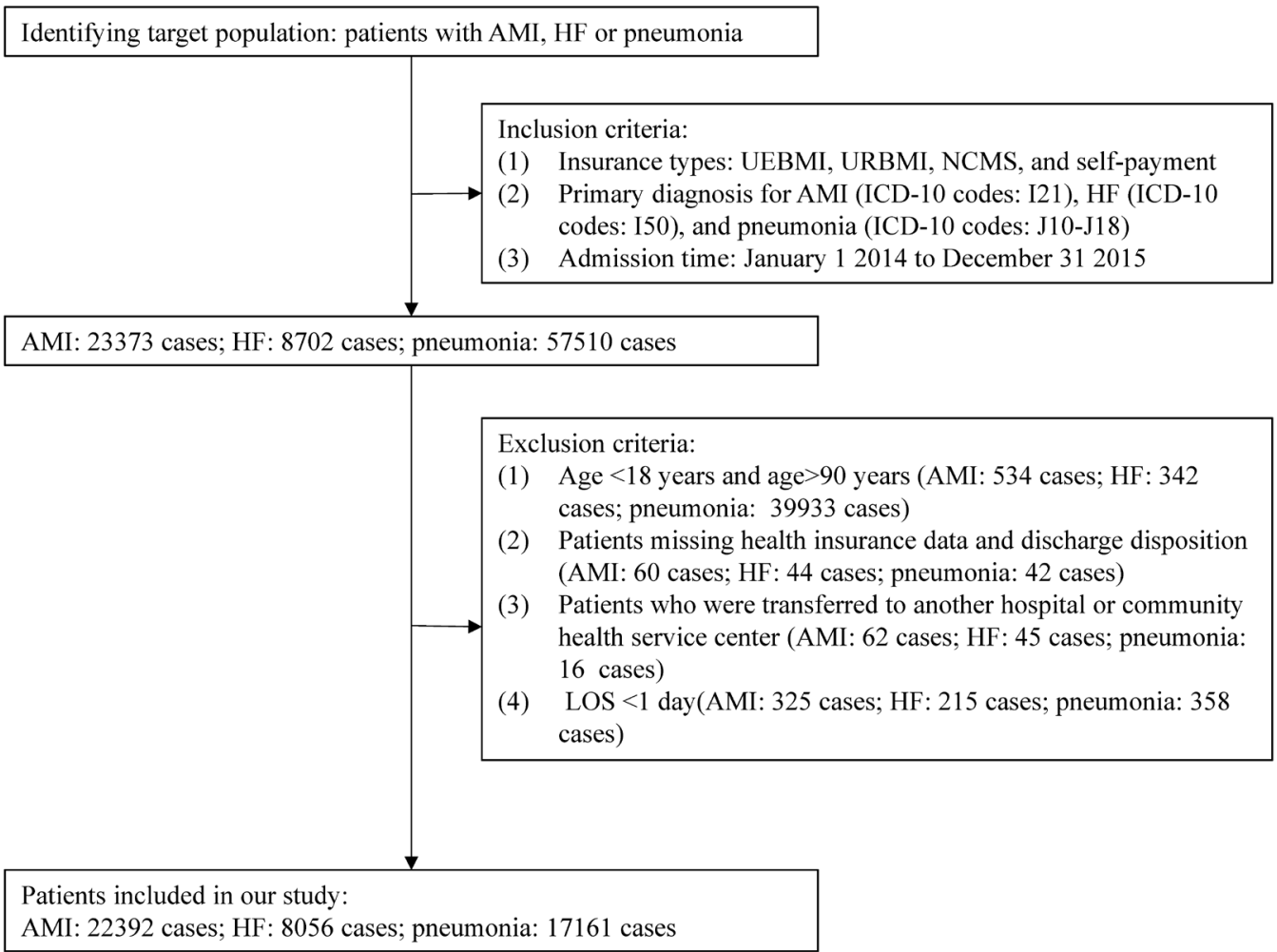

Figure 1 Flow chart of the sampling process. AMI, acute myocardial infarction; HF, heart failure; ICD-10, the international classification of diseases, tenth revision; NCMS, the new cooperative medical scheme; UEBMI, the urban employee-based basic medical insurance; URBMI, the urban resident-based basic medical insurance. 


\section{Study variables}

Inhospital mortality and LOS were selected as the measures of health outcomes during hospitalisation. Inhospital mortality was defined as all deaths that occurred during hospital stay, and LOS was defined as the period from the day of admission to the day of hospital discharge.

The core independent variable was health insurance status, including UEBMI, URBMI and NCMS, as well as self-payment. Previous studies suggested that patients with UEBMI have better outcomes and use more healthcare resources than patients with other insurance types $^{911}{ }^{12}$; hence, UEBMI was selected as the reference group in this study. We selected patient-level and hospital-level covariates based on previous studies to assess the association between health insurance status and health outcomes (adjusted inhospital mortality and LOS). ${ }^{23-26}$ Patient-level covariates included age, gender, admission source, admission condition and comorbid disease. The four categories for admission source are as follows: outpatient medical services, emergency medical services, referral and other sources. The conditions for admission, which is set as a proxy of disease severity, ${ }^{27}$ were classified into three groups, namely, emergency, urgent and regular conditions. Comorbidities were identified from secondary diagnosis, and Charlson Comorbidity Index was considered to assess the effect of comorbid diseases or disorders. ${ }^{28}{ }^{29}$ In this study, the 31 selected tertiary hospitals were teaching hospitals owned by the government. Therefore, we included the following hospital-level covariates: hospital volume, number of hospital beds, number of nurses per 100 beds, number of doctors per 100 beds and hospital region. Hospital volume was defined as the total inpatients who were treated at hospitals using annual condition-specific volume averaged over the 2-year period. For the purpose of characterising the sample, hospitals were categorised into two groups according to the median of hospital volume. The hospital region was classified into three groups, namely, south, north and middle areas.

\section{Statistical analysis}

Patient-level and hospital-level covariates and unadjusted outcomes were compared using ANOVA or Kruskal-Wallis test for continuous variables and either Pearson $\chi^{2}$ analysis or Fisher exact test for categorical variables as appropriate.

We used multilevel mixed-effects logistic regression models to estimate the adjusted effects of health insurance status on inhospital mortality considering the clustering of patients within hospitals. ${ }^{30}$ Multicollinearity was determined using variance inflation factors. The statistical significance of the association between health insurance status and inhospital mortality was assessed via Wald $\chi^{2}$ test. The area under the receiver operating characteristic curve was used to assess statistical model discrimination. Hosmer-Lemeshow test was conducted to evaluate the statistical significance of differences in the calibration of each model among deciles of the observed and predicted risks.

In view of the problems of overdispersion in the model and the clustered effect of patients within the same hospital, we constructed the multilevel mixed-effects negative binomial regression model to estimate the effects of health insurance status on LOS. ${ }^{31}$ The results from this model are in the form of log ratios between the variable and reference groups, which is known as the incidence rate ratio (IRR).

Categorical variables are presented as percentages and continuous variables as means $\pm \mathrm{SD}$. The OR with a $95 \%$ CI and IRRs with a 95\% CI are reported as the results of logistic regression models and negative binomial regression models, respectively. $p$ Values are two tailed. $p<0.05$ was considered statistically significant. All analyses were performed in R software V.3.2.2 and Stata V.14.0 (Stata Crop).

\section{RESULTS}

\section{Patient and hospital characteristics}

In this study, we identified 22392 patients with AMI, 8056 patients with HF and 17161 patients with pneumonia. The frequencies of patient and hospital characteristics stratified on the basis of health insurance status are listed in table 1. Patients with UEBMI (39\%) and NCMS (43\%) represented the largest health insurance group for AMI, HF (UEBMI: 40\%, NCMS: 42\%) and pneumonia (UEBMI: $50 \%$, NCMS: $32 \%$ ). Male gender was the most common characteristic in all of the health insurance groups. Under each condition, the patients in the NCMS group were younger and more likely to be admitted under emergent conditions when they had AMI $(34 \%)$ and HF $(21 \%)$ than the patients in the UEBMI group $(\mathrm{p}<0.001)$. For patients with AMI, $50 \%$ were admitted through outpatient medical services and $48 \%$ through emergency medical services. Meanwhile, this hospitalisation pattern was similar in $\mathrm{HF}$ (77\% and $21 \%$, respectively) and pneumonia (69\% and $22 \%$, respectively). Under each condition, the Charlson Comorbidity Index of the patients with NCMS was lower than that of the UEBMI patients $(\mathrm{p}<0.001)$. A majority of the patients in the self-payment group also received healthcare in hospitals with high bed capacity, and a large proportion of patients sought healthcare in hospitals located in the middle area of Shanxi.

\section{Unadjusted outcomes}

Table 2 presents the unadjusted outcomes of the health insurance groups. The overall inhospital mortality rates for AMI, HF and pneumonia were $4.0 \%, 3.4 \%$ and $7.7 \%$, respectively. Inhospital mortality following pneumonia was highest for NCMS $(11.1 \%)$ and URBMI $(9.1 \%)$ patients. Under each condition, the NCMS patients incurred the highest unadjusted inhospital mortality $(4.7 \%, 4.4 \%$ and $11.1 \%$ for AMI, HF and pneumonia, respectively) among all health insurance groups $(\mathrm{p}<0.001)$, while UEBMI patients yielded the lowest unadjusted inhospital 


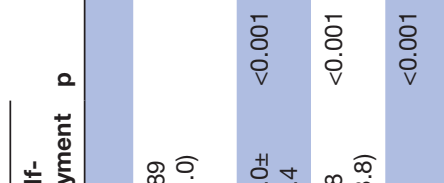

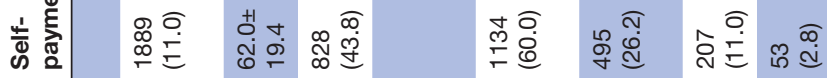

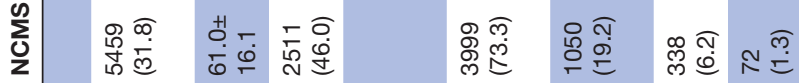

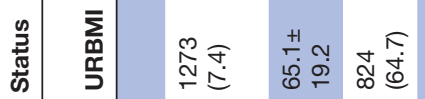

¿

$\frac{2}{<}$

$\frac{2}{4}$

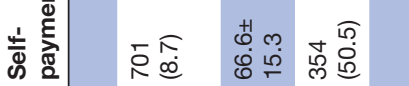

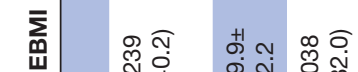

.

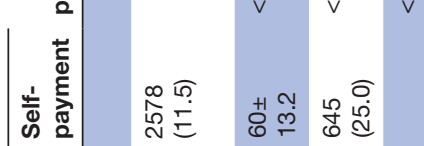

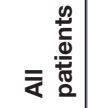

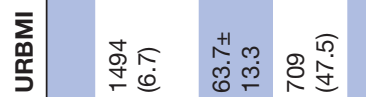

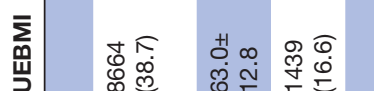

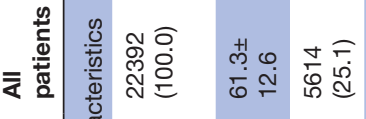

우일

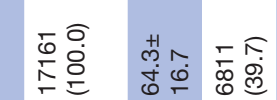

$\begin{array}{lll}\bar{\delta} & \bar{c} & \bar{o} \\ 0 & 0 & 0 \\ \dot{v} & \bar{v} & v\end{array}$

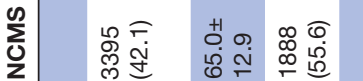

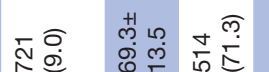

잉

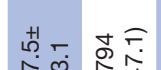

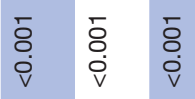

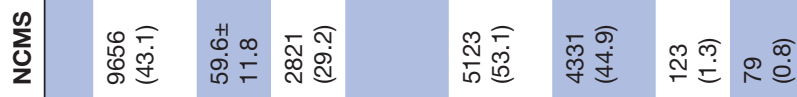

竞离

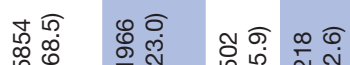

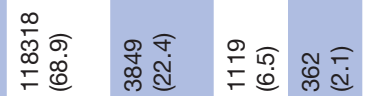

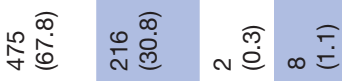

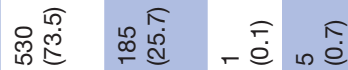

总尽

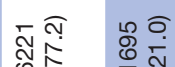

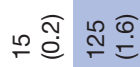

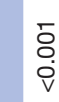

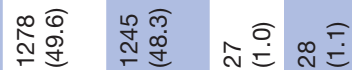

(1)

虽离

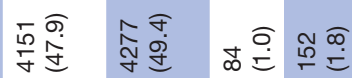

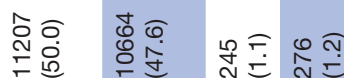

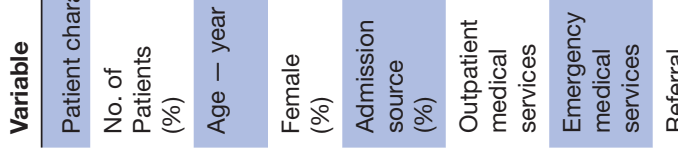

乎离

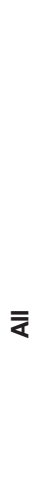

so

$\begin{array}{lll}\overline{8} & \overline{0} & \bar{c} \\ \dot{0} & \dot{0} & \bar{v}\end{array}$

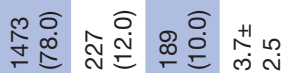

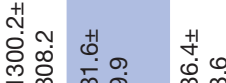

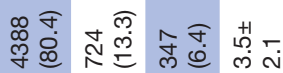

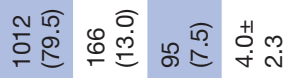

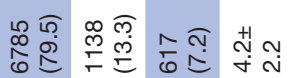

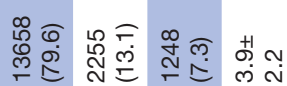

$\overline{8}$
0
0

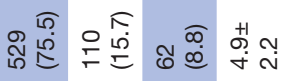

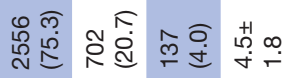

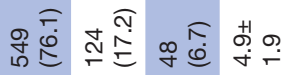

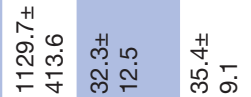

$+1$

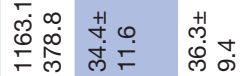

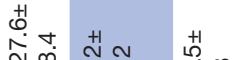

그에

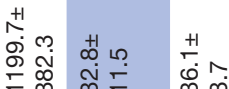

$\begin{array}{lll}\bar{o} & \bar{o} & \bar{o} \\ \dot{0} & \dot{v} & \bar{v}\end{array}$

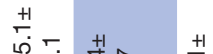

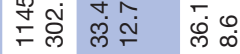

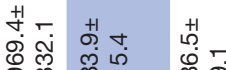

से

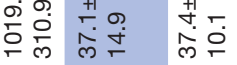

完命

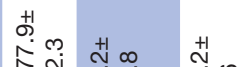

余空

苾

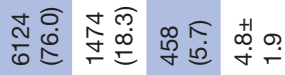

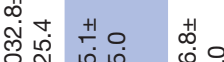

¿্ড.

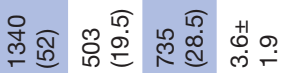

$\begin{array}{lll}\bar{o} & \bar{o} & \bar{o} \\ \dot{0} & \dot{0} & \overline{0} \\ \mathrm{v} & \mathrm{v}\end{array}$

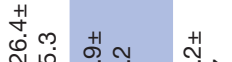

츤

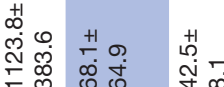

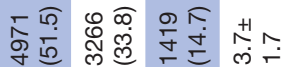

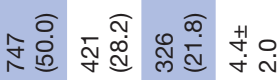

苦 $\infty$

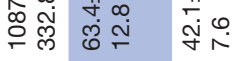

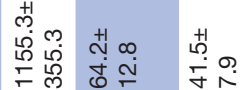

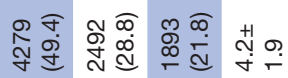

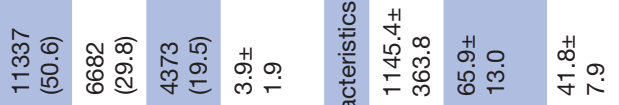




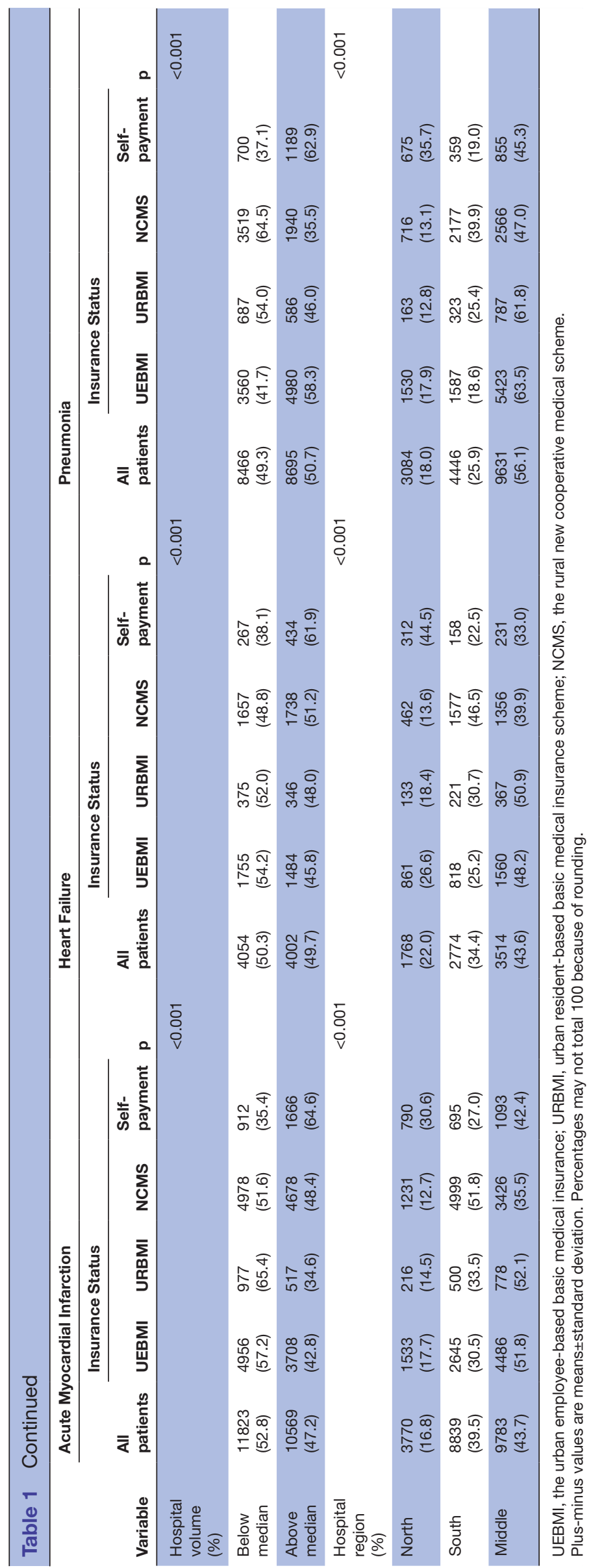

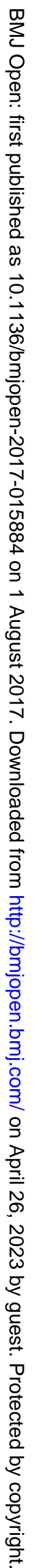


mortality $(3.1 \%, 2.4 \%$ and $5.7 \%$ for AMI, HF and pneumonia, respectively) $(\mathrm{p}<0.001)$.

The mean LOS for AMI, HF and pneumonia were $11.7 \pm 6.6$ days, $10.5 \pm 6.2$ days and $13.0 \pm 9.4$ days, respectively. Under each condition, the patients in the UEBMI group conferred the longest unadjusted mean LOS (12.5 \pm 7.8 days, $11.6 \pm 6.3$ days and $13.9 \pm 9.9$ days for AMI, HF and pneumonia, respectively) among all the health insurance groups followed by URBMI group.

\section{Adjusted outcomes for the effect of health insurance status}

Table 3 shows the results of multilevel multivariable analyses of the relationship between health insurance status and inhospital mortality and LOS after patient-level and hospital-level covariates were controlled.

Adjusted inhospital mortality among AMI patients was significantly higher for NCMS (adjusted OR of 1.39, $95 \% \mathrm{CI}=1.17-1.66)$ and self-payment $(\mathrm{OR}=1.69,95 \% \mathrm{CI}=$ 1.31-2.20), was significantly higher for NCMS among HF patients $(\mathrm{OR}=1.93,95 \% \mathrm{CI}=1.37-2.74)$, and was significantly higher for URBMI (OR=1.64, 95\% CI=1.29-2.10), NCMS (OR=1.97, 95\% CI=1.69-2.30), and self-payment among pneumonia patients $(\mathrm{OR}=1.48,95 \% \mathrm{CI}=1.17-$ $1.87)$ compared with the UEBMI patients. In addition, the results of multilevel mixed-effects negative binomial regression models similarly demonstrated that the LOS of URBMI, NCMS, and self-payment groups under each condition was significantly shorter than that of the UEBMI group $(p<0.001)$. For example, after the patient and hospital characteristics were adjusted in the cases of AMI, our model suggested that the patients in NCMS stayed approximately $90 \%$ of the time in the hospital compared with the patients in UEBMI.

\section{DISCUSSION}

This study sought practical evidence to determine whether there are insurance-related differences in health

\begin{tabular}{|c|c|c|c|c|c|}
\hline Outcome & UEBMI & URBMI & NCMS & Self-payment & $\mathbf{p}$ \\
\hline \multicolumn{6}{|l|}{ AMI } \\
\hline Inhospital mortality (\%) & 3.1 & 4.4 & 4.7 & 4.2 & $<0.001$ \\
\hline LOS (days) & $12.5 \pm 7.8$ & $12.1 \pm 6.1$ & $11.2 \pm 5.6$ & $10.6 \pm 6.0$ & $<0.001$ \\
\hline \multicolumn{6}{|l|}{$\mathrm{HF}$} \\
\hline Inhospital mortality (\%) & 2.4 & 2.8 & 4.4 & 3.9 & $<0.001$ \\
\hline LOS (days) & $11.6 \pm 6.3$ & $10.3 \pm 5.5$ & $9.6 \pm 6.1$ & $10.0 \pm 6.3$ & $<0.001$ \\
\hline \multicolumn{6}{|l|}{ Pneumonia } \\
\hline Inhospital mortality (\%) & 5.7 & 9.1 & 11.1 & 5.7 & $<0.001$ \\
\hline LOS (days) & $13.9 \pm 9.9$ & $13.1 \pm 9.5$ & $11.9 \pm 8.0$ & $11.9 \pm 10.5$ & $<0.001$ \\
\hline
\end{tabular}

AMI, acute myocardial infarction; HF,heart failure; LOS, length of stay; NCMS, the rural new cooperative medical scheme; UEBMI, the urban employee-based basic medical insurance; URBMI, urban resident-based basic medical insurance scheme.

\begin{tabular}{|c|c|c|c|c|c|}
\hline Outcome & UEBMI & URBMI & NCMS & Self-payment & AUC \\
\hline \multicolumn{6}{|l|}{ AMI } \\
\hline Inhospital mortality & 1.00 & 1.22 (0.91 to 1.63$)$ & 1.39 (1.17 to 1.66$)$ & 1.69 (1.31 to 2.20$)$ & 0.80 \\
\hline LOS (days) & 1.00 & $0.96(0.93$ to 0.98$)$ & 0.90 (0.89 to 0.92$)$ & 0.85 (0.83 to 0.87$)$ & - \\
\hline \multicolumn{6}{|l|}{$\mathrm{HF}$} \\
\hline Inhospital mortality & 1.00 & 1.31 (0.76 to 2.27) & 1.93 (1.37 to 2.74$)$ & 1.38 (0.84 to 2.27$)$ & 0.82 \\
\hline LOS (days) & 1.00 & 0.90 (0.87 to 0.94$)$ & 0.85 (0.82 to 0.87$)$ & 0.87 (0.84 to 0.91$)$ & - \\
\hline \multicolumn{6}{|l|}{ Pneumonia } \\
\hline Inhospital mortality & 1.00 & 1.64 (1.29 to 2.10$)$ & 1.97 (1.69 to 2.30$)$ & $1.48(1.17$ to 1.87$)$ & 0.83 \\
\hline LOS (days) & 1.00 & 0.95 (0.92 to 0.99$)$ & 0.87 (0.85 to 0.89$)$ & 0.88 (0.85 to 0.90$)$ & - \\
\hline
\end{tabular}

Inhospital mortality reported as adjusted OR $(95 \% \mathrm{Cl})$. Length of stay reported as incidence rate ratio $(95 \% \mathrm{Cl})$. Reference group: UEBMI. Outcomes adjusted for patient's age, gender, health insurance status, admission source, admission condition, Charlson Comorbidity Index, hospital volume, number of hospital beds (per increases 100 beds), number of nurses per 100 beds, number of doctors per 100 beds and hospital geographical region.

${ }^{*} p<0.1,{ }^{* *} p<0.05,{ }^{* * *} p<0.01$.

AMI, acute myocardial infarction; AUC, area under receiver operator curve; HF,heart failure; LOS, length of stay; NCMS, the rural new cooperative medical scheme; UEBMI, the urban employee-based basic medical insurance; URBMI, urban resident-based basic medical insurance scheme. 
outcomes using administrative data from 31 tertiary hospitals in Shanxi, China, focusing on AMI, HF, and pneumonia. Health insurance status is associated with inhospital mortality and LOS, even after controlling for patient-level and hospital-level covariates. Our results suggested that patients with NCMS have significantly higher risk-adjusted inhospital mortality and shorter LOS in all three conditions than patients with UEBMI, although the association between insurance status and inhospital mortality and LOS varied by diseases in this study.

The impact of health insurance status on health outcomes in China is a recent focus of research. Our results of patients with AMI are consistent with the findings of Liu et al. ${ }^{11}$ Furthermore, our study expanded the limited literature on insurance-related disparities in China in several aspects. First, a broad population comprising patients with AMI, HF and pneumonia was included in our study and thus helped us examine the effects of health insurance status on health outcomes under diverse medical conditions within a large population. Second, the hospital data we used in this study were within 20142015 , which could reflect the situation several years later since China launched its new healthcare reform in 2009. Third, given the cluster effects, we applied the multilevel mixed-effects logistic regression model and the multilevel mixed-effects negative binomial regression model to quantify the effects of health insurance status on inhospital mortality and LOS, respectively. Thus, these models could provide a more exact estimation than conventional logistic regression and linear regression model.

In view of the disparities across health insurance groups, a key question emerges: why are there disparities in health outcomes across health insurance groups, especially between NCMS and UEBMI?

One possible explanation is the wide gap in financial protections. The financial protection of UEBMI is higher than that of NCMS because of greater financing capacity. ${ }^{32} 33$ UEBMI has higher financial protection than NCMS, which may encourage people with UEBMI to consume more healthcare resources, such as having more hospitalisation and longer LOS. An investigation conducted in Zhejiang and Gansu reported that people with UEBMI and URBMI were more likely to seek both inpatient and outpatient care than those with NCMS and with no insurance. ${ }^{34}$ In a study conducted by Ma in $2010,{ }^{35}$ medical insurance predicted the LOS for patients with cerebral infarction, and patients in the medical insurance with higher financial supports might be prone to prolong LOS although more treatments were not required. Personal financial situation also matters apart from the financial support from insurance fund. The attendants who are eligible for UEBMI usually have stable jobs as well as high income level, ${ }^{9}$ and their payment capacity to high-priced treatments or medications that may improve their health outcomes is stronger than NCMS participants. Furthermore, from the perspective of health providers, they may be vigilant regarding new and expensive treatment forms and even provide different treatments for patients with the same condition but with different insurance status. ${ }^{36}$ Patients with higher levels of payments may be given higher priority to receive healthcare services. $^{37}$

Another possible explanation is the wide gap in benefit coverage across insurance schemes. As reported by the WHO, the SHI schemes in China vary largely in the following dimensions: breadth (percentage of population covered), depth (percentage of health costs covered) and scope (type of health services covered) ${ }^{38}$ NCMS covered more population than the UEBMI, but it was inferior in terms of depth and scope, and NCMS participants possess lower reimbursement level and smaller service coverage ${ }^{39-41}$ In 2011, the reimbursement rate of inpatient care for NCMS was $44 \%$, while it was $68 \%$ for UEBMI participants. ${ }^{3}$ In addition, UEBMI patients have more comprehensive services coverage than NCMS patients, with the expenses of some medical service items reimbursed to patients with UEBMI rather than patients with NCMS. The lower reimbursement level and more limited services coverage mean higher out-of-pocket expenses to patients with NCMS and may influence their choice of treatments and time to surgery, eventually resulting in the significant differences of health outcomes. For instance, dual antiplatelet therapy (DAPT) is an essential therapy for patients who received reperfusion therapy, while it means heavy economic burden to some NCMS patients because of its high cost and low reimbursement percentage. ${ }^{11}$ Therefore, DAPT adherence was lower in the NCMS group than in other health insurance groups, which finally lead to higher inhospital mortality. Additionally, NCMS patients tend to take medical cost as a priority when they decide whether to receive treatment or not, which may cause treatment delay and eventually affect the prognosis of patients. ${ }^{11}$ Other possible explanations, such as the experience of physicians, differences in procedures, education level and lifestyle, accounting for the differences in health insurance status have been suggested by relevant studies, ${ }^{42-44}$ but empirical evidence in China remains insufficient.

Calls for the consolidation of SHI schemes towards an equitable and efficient system have increased in recent years since the SHI schemes were criticised for its fragmentation, ${ }^{45-47}$ but the progress of the consolidation is slow. Several provinces or municipalities have piloted the consolidation of these schemes, and some evidence showed that the consolidation contributed to the equitable access to healthcare and efficiency of the system. ${ }^{45}$ In early 2016, the State Council of People's Republic of China promulgated a guideline on the integration of the URBMI and NCMS, which aims to create a unified basic health insurance system. The URBMI and NCMS would be unified in six areas, namely, insurance coverage, insurance finance, payment standard, medicine and medical service item catalogue, management of the qualified insurance institutions and insurance accounting. ${ }^{48}$ This policy guideline has promoted the consolidation of SHI 
schemes in China, which provides an alternative way to narrow the gaps between different insurance programs and improve health equity. However, the consolidation may take a long process considering the lack of specific matching plans or policies, difficulty in merging administrative institutions and staff, unifying funding level and benefit packages, reforming payment systems and strengthening information systems. ${ }^{36-45}$

\section{Policy implications}

The universal health insurance coverage in China has improved the accessibility to healthcare services, but the government has paid little attention to the disparities in health outcomes among health insurances. This study reveals the disparities in inhospital mortality and LOS across different insurance schemes. We suggest that policies be made to narrow the gaps of insurance benefits for patients with AMI, HF or pneumonia, such as increasing reimbursement rate for NCMS and URBMI, expanding the service coverage for NCMS and URBMI and consolidating SHI schemes. On the other hand, policy-makers should adjust the reimbursement policies of some expensive treatments according to the results of clinical practice and economic assessment, such as increasing the reimbursement rate of DAPT for NCMS patients with AMI.

\section{Limitations}

This study has several limitations. First, although our study suggested the association between health insurance status and health outcomes, we are unable to identify a causal relation because of the cross-sectional research design. Second, the three common conditions we analysed represent a large proportion of hospitalisations in Shanxi; however, the results of these data were difficult to generalise into the full spectrum of hospitalisations or patients in other levels of hospitals in China. Finally, capturing all potential confounders in our study is difficult, although we anticipated many challenges in data collection. Some details on treatment information and individual socioeconomic status that might be related to health outcomes, such as medications, employment, income and education, were not available for this study. Therefore, these data were not included in our analysis despite such information is an important indicator of healthcare quality. However, we collected the patient and hospital characteristics adopted by previous studies in our database as much as possible and used standard and well-accepted risk-adjustment techniques to reduce potential bias from the unmeasured confounders.

\section{Further research}

The observed disparities in our study reveal the potential influence of insurance status on health outcomes in inpatient service practices in Shanxi, China. However, the underlying mechanisms of the association between insurance status and health outcomes are complicated. Further research is necessary to explore the underlying mechanisms and validate this relationship under other diagnoses, such as cardiovascular surgery, orthopaedic surgery and tumour therapy. Moreover, future studies focusing on various dimensions of quality, including process of care and the experience of patients, are warranted.

\section{CONCLUSIONS}

Health insurance status is associated with the health outcomes of patients who were hospitalised for AMI, HF and pneumonia in Shanxi, China. In particular, the adjusted inhospital mortality rate of NCMS patients was significantly higher, and their LOS was shorter than that of UEBMI patients. Further research should be conducted to understand the mechanisms of the effects of health insurance status on health outcomes to support policy formulation and implementation. Policies also should be considered and formulated by the government to minimise the gaps across different insurance schemes and further improve the equity of healthcare delivery in China.

Acknowledgements The authors sincerely thank the Health and Family Planning Commission in Shanxi for providing us with the data used in this study. The authors thank China Scholarship Council for supporting to communicate this study in Saint Louis University. We also thank Shanquan Chen for his generous comments on interpretation of the results of this article. KGSupport, a professional copyediting agency, helped to further refine manuscript to fulfil the high standards required for publication.

Contributors XL researched the data, performed the statistical analysis and wrote the manuscript. XL, MC, $\mathrm{HT}$ and $\mathrm{EL}$ contributed to the interpretation of the data and the discussion of the manuscript. ZC, CX, MW, SX and TJ contributed to the data acquisition and provided statistical analysis support. All the authors supplied critical revisions to the manuscript and gave final approval of the version to be published.

Funding This work was supported by the National Natural Science Foundation of China (grant number 71473099).

Competing interests None declared.

Ethics approval This project was reviewed and approved by the Ethics Committee of Tongji Medical College, Huazhong University of Science and Technology (IORG No.: IORG0003571).

Provenance and peer review Not commissioned; externally peer reviewed. Data sharing statement No additional data are available.

Open Access This is an Open Access article distributed in accordance with the Creative Commons Attribution Non Commercial (CC BY-NC 4.0) license, which permits others to distribute, remix, adapt, build upon this work non-commercially, and license their derivative works on different terms, provided the original work is properly cited and the use is non-commercial. See: http://creativecommons.org/ licenses/by-nc/4.0/

(c) Article author(s) (or their employer(s) unless otherwise stated in the text of the article) 2017. All rights reserved. No commercial use is permitted unless otherwise expressly granted.

\section{REFERENCES}

1. Yip WC, Hsiao WC, Chen W, et al. Early appraisal of China's huge and complex health-care reforms. Lancet 2012;379:833-42.

2. Tang S, Brixi H, Bekedam H. Advancing universal coverage of healthcare in China: translating political will into policy and practice. Int J Health Plann Manage 2014;29:160-74.

3. Meng Q, Xu L, Zhang Y, et al. Trends in access to health services and financial protection in China between 2003 and 2011: a crosssectional study. Lancet 2012;379:805-14.

4. Liang L, Langenbrunner J. The long march to universal coverage: lessons from China. Washington, DC: World Bank, 2013:5.

5. Dong K. Medical insurance system evolution in China. China Economic Review 2009;20:591-7.

6. National Health and Family Planning Commission of the People's Republic of China. 2016 China statistical yearbook of health and family planning. Beijing: Peking Union Medical College Press, 2016. 
7. Department of Population and Employment Statistics of National Bureau of Statistics, Department of Planning and Finance of Ministry of Human Resource and Social Security. China Labour Statistical Yearbook 2015. Beijing: China Statistics Press, 2015.

8. Sun Y, Gregersen H, Yuan W. Chinese health care system and clinical epidemiology. Clin Epidemiol 2017;9:167-78.

9. Liu X, Wong H, Liu K. Outcome-based health equity across different social health insurance schemes for the elderly in China. BMC Health Serv Res 2015;16:1-12

10. Yu BR. Influences of health insurance status on clinical treatments and outcomes for 4,714 patients after acute myocardial infarction in 14 chinese general hospitals. J Med Dent Sci 2005;52:143-51.

11. Liu B, Yan H, Guo R, et al. The basic social medical insurance is associated with clinical outcomes in the patients with ST-elevation myocardial infarction: a retrospective study from Shanghai, China. Int $J$ Med Sci 2014;11:905-17.

12. Wang Z, Zhang $Y$, Xiong F, et al. Association between medical insurance type and survival in patients undergoing peritoneal Dialysis. BMC Nephrol 2015;16:33.

13. Kong $\mathrm{Y}$, Wang $\mathrm{Y}$, Zhang JH, et al. Disparities in medical expenditure and outcomes among patients with intracranial hemorrhage associated with different insurance statuses in southwestern China. Acta Neurochir Suppl 2011:111:337-41.

14. Feng $Y, X i o n g X, X u e ~ Q$, et al. The impact of medical insurance policies on the hospitalization services utilization of people with schizophrenia: a case study in Changsha, China. Pak J Med Sci 2013;29:793-8

15. Fang $\mathrm{H}$, Meng Q, Rizzo JA. Do different health insurance plans in China create disparities in health care utilization and expenditures? International Journal of Applied Economics 2014;11:1-18.

16. The Bureau of Statistics in Shanxi, China. Shanxi Statistical Yearbook. Beijing: China Statistics Press, 2015.

17. National Center for Cardiovascular Disease, China. Report on cardiovascular disease in China 2015. Beijing: Encyclopedia of China Publishing House, 2016.

18. Jiang $\mathrm{H}, \mathrm{Ge} J$. Epidemiology and clinical management of cardiomyopathies and heart failure in China. Heart 2009;95:1727-31.

19. Xu H, Li W, Yang J, et al. The China Acute Myocardial Infarction (CAMI) Registry: a national long-term registry-research-education integrated platform for exploring acute myocardial infarction in China. Am Heart J 2016;175:193-201.

20. National Center for Cardiovascular Disease, China. Report on cardiovascular disease in China 2014. Beijing: Encyclopedia of China Publishing House, 2015.

21. He J, Gu D, Wu X, et al. Major causes of death among men and women in China. N Engl J Med 2005;353:1124-34

22. He L, Chen X. Contentions on the pathogenic spectrum of community-acquired pneumonia and the first experimental application of antibiotic agents. Chinese Journal of Practical Internal Medicine 2007;27:110-3.

23. Hasan O, Orav EJ, Hicks LS. Insurance status and hospital care for myocardial infarction, stroke, and pneumonia. $J$ Hosp Med 2010;5:452-9.

24. Kim JH, Lee SG, Lee KS, et al. Impact of health insurance status changes on healthcare utilisation patterns: a longitudinal cohort study in South Korea. BMJ Open 2016;6:e9538.

25. LaPar DJ, Bhamidipati CM, Mery CM, et al. Primary payer status affects mortality for Major surgical operations. Ann Surg 2010;252:148-56

26. Spencer CS, Gaskin DJ, Roberts ET. The quality of care delivered to patients within the same hospital varies by insurance type. Health Aff 2013;32:1731-9.

27. Xu Y, Liu Y, Shu T, et al. Variations in the quality of care at large public hospitals in Beijing, China: a condition-based outcome approach. PLoS One 2015;10:e138948.
28. Quan $\mathrm{H}$, Sundararajan $\mathrm{V}$, Halfon $\mathrm{P}$, et al. Coding algorithms for defining comorbidities in ICD-9-CM and ICD-10 administrative data. Med Care 2005;43:1130-9.

29. Charlson ME, Pompei P, Ales KL, et al. A new method of classifying prognostic comorbidity in longitudinal studies: development and validation. J Chronic Dis 1987;40:373-83.

30. Rice N, Leyland A. Multilevel models: applications to health data. $J$ Health Serv Res Policy 1996;1:154-64.

31. Carter EM, Potts HW. Predicting length of stay from an electronic patient record system: a primary total knee replacement example. BMC Med Inform Decis Mak 2014;14:26.

32. Liu K, Wu Q, Liu J. Examining the association between social health insurance participation and patients' out-of-pocket payments in China: the role of institutional arrangement. Soc Sci Med 2014;113:95-103.

33. Yu H. Universal health insurance coverage for 1.3 billion people: what accounts for China's success? Health Policy 2015;119:1145-52.

34. Li X, Zhang W. The impacts of health insurance on health care utilization among the older people in China. Soc Sci Med 2013;85:59-65.

35. Ma Y, Liu Y, Fu HM, et al. Evaluation of admission characteristics, hospital length of stay and costs for cerebral infarction in a mediumsized city in China. Eur J Neurol 2010:17:1270-6.

36. He AJ, Wu S. Towards universal health coverage via social health insurance in China: Systemic fragmentation, reform imperatives, and policy alternatives. Appl Health Econ Health Policy 2016:1-10.

37. Zhong $\mathrm{H}$. Effect of patient reimbursement method on health-care utilization: evidence from China. Health Econ 2011;20:1312-29.

38. World Health Organization. Sustainable health financing, universal coverage and social health insurance. World Health Assembly Resolution, 2005:58.

39. Fu R, Wang $\mathrm{Y}, \mathrm{Bao} \mathrm{H}$, et al. Trend of urban-rural disparities in hospital admissions and medical expenditure in China from 2003 to 2011. PLoS One 2014:9:e1085719.

40. Wagstaff $A$, Lindelow M, Jun G, et al. Extending health insurance to the rural population: an impact evaluation of China's new cooperative medical scheme. J Health Econ 2009;28:1-19.

41. Barber SL, Yao L. Development and status of health insurance systems in China. Int J Health Plann Manage 2011;26:339-56.

42. Canto JG, Rogers WJ, French WJ, et al. Payer status and the utilization of hospital resources in acute myocardial infarction: a report from the National Registry of myocardial infarction 2. Arch Intern Med 2000;160:817-23.

43. Mainous AG, Diaz VA, Everett CJ, et al. Impact of insurance and hospital ownership on hospital length of stay among patients with ambulatory care-sensitive conditions. Ann Fam Med 2011;9:489-95.

44. Lantz PM, House JS, Lepkowski JM, et al. Socioeconomic factors, health behaviors, and mortality: results from a nationally representative prospective study of US adults. JAMA 1998;279:1703-8.

45. Meng Q, Fang $\mathrm{H}$, Liu X, et al. Consolidating the social health insurance schemes in China: towards an equitable and efficient health system. Lancet 2015;386:1484-92.

46. Wang HQ, Liu ZH, Zhang YZ, et al. Integration of current identitybased district-varied health insurance schemes in China: implications and challenges. Front Med 2012;6:79-84.

47. Wang X, Zheng A, He X, et al. Integration of rural and urban healthcare insurance schemes in China: an empirical research. BMC Health Serv Res 2014;14:142.

48. State Council of People's Republic of China. Guideline for integration of basic medical insurance for urban employees and the new rural cooperative medical scheme. 2016. http://www.gov.cn/ zhengce/content/2016-01/12/content_10582.htm. (accessed 22 Sep 2016). 